\title{
Preparation of polymer gold nanoparticle composites with tunable plasmon coupling and their application as SERS substrates
}

\author{
Samir A. Belhout, Frederico R. Baptista, Stephen J. Devereux, \\ Anthony W. Parker, Andrew D. Ward and Susan J. Quinn
}

\section{Published version information}

Citation: SA Belhout et al. "Preparation of polymer gold nanoparticle composites with tunable plasmon coupling and their application as SERS substrates." Nanoscale vol. 11, no. 42 (2019): 19884-19894.

\section{DOI: $\underline{10.1039 / c 9 n r 05014 k}$}

This version is made available in accordance with publisher policies. Please cite only the published version using the reference above. This is the citation assigned by the publisher at the time of issuing the AAM. Please check the publisher's website for any updates. 


\section{Preparation of polymer gold nanoparticle composites with tunable plasmon coupling and their application as SERS substrates}

Received 00th January 20xx, Accepted 00th January 20xx DOI: $10.1039 / \times 0 \times x 00000 x$

\begin{abstract}
Samir A. Belhout, ${ }^{a}$ Frederico R. Baptista, ${ }^{a}$ Stephen J. Devereux, ${ }^{a}$ Anthony W. Parker, ${ }^{b}$ Andrew D. Ward ${ }^{\mathrm{b}}$ and Susan J. Quinn*a

The controlled surface functionalisation of polystyrene beads (200 nm) with a lipoic acid derivative is used to assemble composites with between 4 to $20 \%$ loadings of citrate stabilised gold nanoparticle ( $13 \mathrm{~nm}-30 \mathrm{~nm}$ ), which exhibit variable optical properties arising from interaction of the nanoparticle surface plasmon resonance (SPR). The decrease in average interparticle distance at higher loadings results in a red-shift in the SPR wavelength, which is well described by a universal ruler equation. The composite particles are shown to act as good SERS substrates for the standard analyte 4mercaptophenol. The direct assessment of the SERS activity for individual composite particles solution is achieved by Raman optical tweezer measurements on $5.3 \mu \mathrm{m}$ composite particles. These measurements show an increase in performance with increasing AuNP size. Importantly, the SERS activity of the individual particles compares well with the bulk measurements of samples deposited on a surface, indicating that the SERS activity arises primarily from the composite and not due to composite-composite interactions. In both studies the optimum SERS response is obtained with $30 \mathrm{~nm}$ AuNPs.
\end{abstract}

\section{Introduction}

Metal nanoparticles are widely studied due to their unique properties that differ from bulk materials; ${ }^{1}$ notably, their size dependent optical properties that arise from the uniform oscillation of the NP's conductance band electrons, termed the surface plasmon resonance (SPR). ${ }^{2}$ The SPR band is influenced by the size, ${ }^{3}$ shape $^{4}$ and composition ${ }^{5}$ of the NP, as well as the refractive index of the surrounding media, which is described through a number of models.6,7 Gold nanoparticles (AuNPs) are particularly well studied and applications include plasmon enhanced catalysis, ${ }^{8}$ electrocatalytic activity, ${ }^{9}$ sensing, ${ }^{10}$ payload delivery, ${ }^{11}$ and radiosensitisation. ${ }^{12}$ In order to preserve the optical properties of AuNPs, it is important to ensure the sample stability and resistance to aggregation ${ }^{13}$ One approach to achieve this is to use AuNP composite materials, ${ }^{14}$ which can be prepared through in-situ growth ${ }^{15}$ or the immobilisation of pre-formed AuNPs on a supporting material. ${ }^{16}$ In the latter case a variety of supports have been used including polymer beads, ${ }^{17}$ silica, ${ }^{13,18,19}$ nanotubes ${ }^{20}$ and graphene oxide. ${ }^{21}$ These materials have been shown to display improved stability to biological medium ${ }^{14}$ and improved performance compared to discrete AuNPs, particularly in surface enhanced Raman spectroscopy (SERS). ${ }^{22}$

\footnotetext{
a. School of Chemistry, University College Dublin, Dublin 4, Republic of Ireland ${ }^{b}$ Central Laser Facility, Research Complex at Harwell, Email: susan.quinn@ucd.ie

Central Laser Facility, Research Complex at Harwell, STFC Rutherford Appleton Laboratory, Harwell Oxford, Didcot, Oxfordshire, OX11 OQX, UK

Electronic Supplementary Information (ESI) available: [details of any supplementary information available should be included here]. See DOI: 10.1039/x0xx00000x
}

SERS activity is related to coupling of the SPR of adjacent AuNPs, which leads to the generation of plasmonic 'hot spots' areas of large electromagnetic enhancement between the AuNPs. ${ }^{23,24}$ These hot spots have been shown to give extremely high enhancement in the Raman response that correlates with both the hot spot size $\mathrm{e}^{25}$ and the plasmonic response ${ }^{26}$ of the coupled AuNPs. The ability to tune the plasmonic response for spherical and isotropic gold spherical nanoparticles has received significant attention. ${ }^{27,28}$ SPR coupling (hybridisation) of adjacent AuNPs results in a redshift in the SPR band, which $^{29,30}$ is directly related to the AuNP separation ${ }^{31,32}$ with negligible effects at separations greater than 1.5 times the nanoparticle diameter. ${ }^{33}$ This universal behaviour has been described by an empirically determined ruler equation for coupled pairs of $\mathrm{Au}$ discs ${ }^{31}$ and spherical AuNPs. ${ }^{34,35}$ The ruler equation also holds-up in the case of more complex systems such as 2D arrays of $20-100 \mathrm{~nm}$ Au spherical NPs ${ }^{36}$ and linear chains of $10 \mathrm{~nm}$ AuNPs. ${ }^{37}$ While, related equations have been developed for dimers of gold nanocubes, ${ }^{33}$ gold mushrooms ${ }^{38}$ and silver nanodisks. ${ }^{33}$ Thus, although empirical, these plasmon scaling equations have potential to act as a useful tool for the design of nanostructured plasmonic materials.

The ability to control the interparticle distance at the surface of composite materials offers the prospect to tune the NP optical properties for applications such as $\mathrm{SERS}^{39,40}$ and plasmonic catalysis. ${ }^{41}$ Yet while precise control can be gained by lithographically patterning on surfaces, ${ }^{35}$ it is significantly more challenging to achieve this at the surface of dispersed support particles. Previous attempts to control the extent of AuNP coverage at a substrate surface have exploited the ratio of AuNP to the support NP,14,42 and also recognised the role of electrostatic repulsion in limiting the overall loading. ${ }^{19,43}$ In 
particular, polymer based materials have proven to be versatile substrates for the preparation of SERS nanocomposites. ${ }^{44}$ Polydopamine nanoparticles have recently been used as supports for the in-situ growth of concentric Au nanoshells and AuNP multilayer systems. ${ }^{45,46}$ The use of Au nanostar-coated polystyrene (PS) beads as multifunctional SERS probes have also been reported. ${ }^{22}$ In addition, we recently showed that polystyrene supported AuNP composites have excellent colloidal stability in buffered solution and in cell medium at physiological temperature. ${ }^{14}$ Our current study reports the functionalisation of $200 \mathrm{~nm}$ polystyrene (PS) particles with different amounts of a lipoic acid derived anchoring ligand (1), which has a known affinity for the AuNP surface, to prepare PSLA particles. In the absence of 1 the PS particle shows no affinity for the AuNPs. However, using the PSLA particles families of AuNP@PS composite materials with tunable surface coverage of different sized AuNPs can be prepared, Scheme 1. In contrast to previous core-shell polymer nanocomposite systems, ${ }^{45,46}$ our approach has the advantage of providing control of the population of size defined AuNPs immobilised at the surface of the bead, and as a consequence, the AuNP separations at the surface. An excellent correlation between the increased population of ligand $\mathbf{1}$ and the increased AuNP loading is found, and the influence of the AuNP coupling on the SPR band of the composite materials is examined. The resulting plasmonic properties were found to be well described by a plasmon ruler equation. Furthermore, a correlation between plasmonic behaviour of the AuNPs on the surface to their SERS activity is explored.

Optical trapping Raman microscopy is a powerful tool for the study of chemical processes and transformations, and for the study of dilute particle solutions whose Raman spectra are otherwise difficult to obtain. ${ }^{47}$ Optical trapping has been used to monitor changes in the heme vibrations within a trapped blood cell upon changing solution $\left[\mathrm{O}_{2}\right], 48$ and changes in phospholipid membrane structure. ${ }^{49}$ It has also recently been

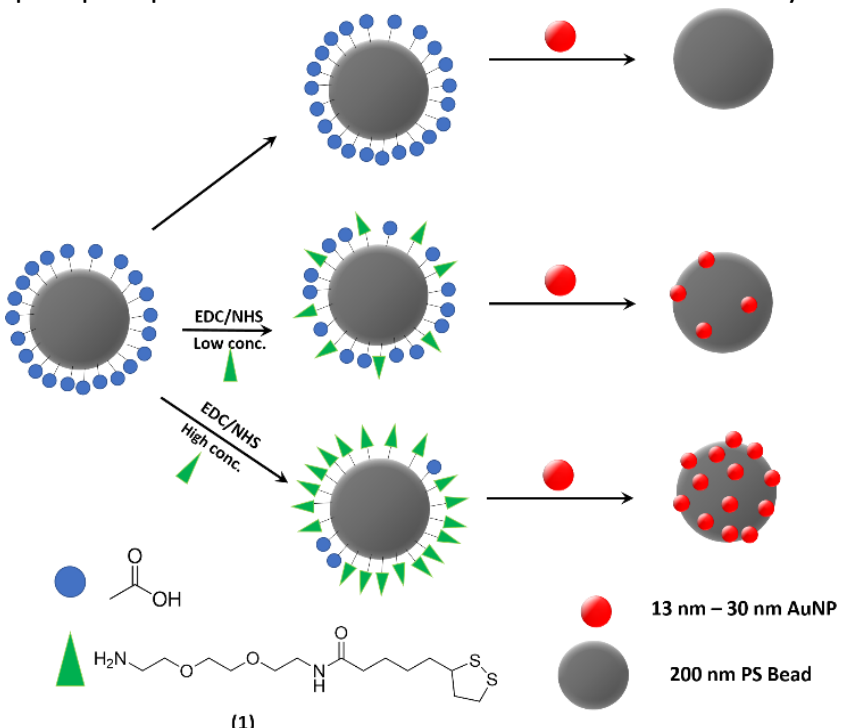

(1)

Scheme 1. Schematic representation of tunable AuNP immobilisation onto PS beads through availability of reactive surface groups. used to probe the SERS activity of metal activated particles. ${ }^{50}$ In the final part of this study we examine the use of Raman optical tweezers to investigate the SERS activity of individual composites to 4-mercaptophenol (4-MP). These results are compared to those of a bulk deposited sample and provide important insights into the ability to translate the discrete properties to further application.

\section{Experimental}

\section{Materials}

Lipoic acid, gold(III) chloride, trisodium citrate, hydrochloric acid $(\mathrm{HCl})$, nitric acid $\left(\mathrm{HNO}_{3}\right)$ sodium chloride $(\mathrm{NaCl})$, phosphate buffered saline tablets (PBS), N-(3-Dimethylaminopropyl)- $\mathrm{N}^{\prime}$ ethylcarbodiimide hydrochloride (EDC), $\mathrm{N}$-hydroxysuccinimide (NHS) and 4-mercaptophenol (4-MP) were all supplied from Sigma Aldrich. 1,8-Diamino-3,6-dioxaoctane was supplied by Merck. $200 \mathrm{~nm}$ and $5.3 \mu \mathrm{m}$ carboxylate-modified polystyrene beads (PS- $\mathrm{COOH}$ ) were supplied by ThermoFisher. All materials were analytical grade and were used without any further purification. All water was obtained from an ultra-pure Millipore $0.22 \mu \mathrm{m}$ filtration system (18.2 $\mathrm{m} \Omega$ conductivity).

\section{Characterisation}

UV-visible (UV-vis) spectroscopy (Varian Cary-50 Eclipse spectrophotometer) was used to characterize the assynthesised AuNPs as well as the composite materials. Atomic absorption spectroscopy (AAS) (Varian SpectrAA 55B atomic absorption spectrometer) was used to determine the quantity of AuNPs on the surface of the composite materials. Dynamic light scattering (DLS) and $\zeta$-potential measurements were carried out on a Malvern Zetasizer Nano-ZS. Agitation was performed using a Grant-bio PCMT Thermo shaker. Centrifugation was carried out using a Thermo Scientific Heraeus pico 17 centrifuge. Sonication was carried out using a Branson 1510 sonic bath $(42 \mathrm{kHz}, 80 \mathrm{~W})$. Scanning electron microscopy (SEM) was performed using a Zeiss ULTRA plus field emission microscope using the secondary electron detector at $5 \mathrm{kV}$ acceleration.

\section{Synthesis of lipoic amine molecule $(1)^{51,52}$}

Tert-butyl(2-(2-(2-(5-(1,2-dithiolanyl)pentanamido)ethoxy) ethoxy)ethoxy)ethyl)carbamate was prepared according to previous literature methods and is detailed in the ESI.

\section{Controlled functionalisation of $200 \mathrm{~nm}$ PS-COOH with 1}

The surface - $\mathrm{COOH}$ occupancy of $200 \mathrm{~nm}$ PS-COOH was taken from supplier (lot \#1845148, $9.5 \times 10^{4}$ carboxyl groups (1.59 x $10^{-19} \mathrm{~mol}$ ) per particle). $75 \mu \mathrm{L}$ of PS-COOH $(0.12 \mu \mathrm{mol}$ surface $\mathrm{COOH}$ groups) was added to a $2 \mathrm{~mL}$ Eppendorf tube. To this, aqueous solutions of EDC $(200 \mu \mathrm{L}, 78.2 \mathrm{mM})$ and NHS $(200 \mu \mathrm{L}$, $130 \mathrm{mM}$ ) were added. The mixture was agitated at $1400 \mathrm{rpm}$ at $25{ }^{\circ} \mathrm{C}$ for $2 \mathrm{~h}$ afterwhich $1000 \mu \mathrm{L}$ of an aqueous solution of 1 $(0.055 \mu \mathrm{mol}-3.3 \mu \mathrm{mol})$ was added and agitated for $5 \mathrm{~h}$ (PSLA1, 
$0.055 \mu \mathrm{mol}$ lipoic amine or $16 \mathrm{~h}$ (PSLA2-4, $0.055-3.3 \mu \mathrm{mol}$ (1)). Samples were washed by centrifugation to remove excess EDC, NHS and lipoic amine. Modified PS beads were analysed by $\zeta$-potential and DLS and stored in Millipore $\mathrm{H}_{2} \mathrm{O}$ at RT.

\section{Functionalisation of $5.3 \mu \mathrm{m}$ PS-COOH with 1}

Occupancy of - $\mathrm{COOH}$ groups on the surface of the $5.3 \mu \mathrm{m}$ beads was determined using lot data (lot \#1833760, $1.5 \times 10^{9}$ carboxyl groups $\left(2.49 \times 10^{-19} \mathrm{~mol}\right)$ per particle) provided by the supplier. $600 \mu \mathrm{L}$ of PS- $\mathrm{COOH}(0.12 \mu \mathrm{mol}$ surface $-\mathrm{COOH}$ groups) was added to a $2 \mathrm{~mL}$ Eppendorf tube and the protocol described above for $200 \mathrm{~nm}$ PSLA4 was repeated (16 h, $3.3 \mu \mathrm{mol}$ (1) $(5.3$ $\mu \mathrm{m}$ PSLA4).

\section{Preparation of $13-30 \mathrm{~nm}$ gold nanoparticles}

AuNPs of different sizes were prepared via the seeded growth method developed by Puntes et al.. ${ }^{53}$ Briefly, a seed solution was prepared by injecting $1 \mathrm{~mL}$ of chloroauric acid solution ( 25 $\mathrm{mM}$ ) into $150 \mathrm{~mL}$ of a boiling solution of sodium citrate (2.2 $\mathrm{mM}$ ) contained in a 3-neck round bottom flask and fitted with a reflux condenser and thermometer. The reaction was allowed to proceed for $15 \mathrm{~min}$ and then cooled to $90^{\circ} \mathrm{C}$. Chloroauric acid solution ( $1 \mathrm{~mL}, 25 \mathrm{mM}$ ) was injected into the seed solution and allowed to react for $30 \mathrm{~min}$. This was repeated twice. After the third addition had reacted for $30 \mathrm{~min}, 55 \mathrm{~mL}$ of sample was harvested and replaced with $53 \mathrm{~mL}$ of Millipore $\mathrm{H}_{2} \mathrm{O}$ and $2 \mathrm{~mL}$ of sodium citrate solution $(60 \mathrm{mM})$. This acted as the seed solution for the next generation. This process was repeated five times. The synthesized gold nanoparticles were characterized using UV-vis, AAS, DLS and TEM.

\section{General procedure for composite formation}

Constant concentrations of different sized AuNPs (10 nm - 30 $\mathrm{nm}, 1 \mathrm{~mL}$ as-prepared stock solution) were added to a $1 \mathrm{~mL}$ aqueous solution containing support materials at constant concentrations (see ESI, Table S1) The samples were agitated at $1000 \mathrm{rpm}$ for $60 \mathrm{~min}$ at $25^{\circ} \mathrm{C}$. The samples were then centrifuged $(1200 \mathrm{rpm}$ - $30 \mathrm{~nm}$ AuNPs, $1500 \mathrm{rpm}-26 \mathrm{~nm}$ AuNPs, $2000 \mathrm{rpm}-22 \mathrm{~nm}$ AuNPs, $2800 \mathrm{rpm}-17 \mathrm{~nm}$ AuNPs, $3300 \mathrm{rpm}-13 \mathrm{~nm}$ AuNPs, $20 \mathrm{~min}$ ), to pellet any composite material and leave free AuNPs in the supernatant. The supernatant was removed and analysed by UV-vis and AAS. The composites were repeatedly washed to remove any free AuNPs and finally re-dispersed in $1 \mathrm{~mL}$ Millipore $\mathrm{H}_{2} \mathrm{O}$ for analysis.

\section{Determination of average AuNP-AuNP separation on the $200 \mathrm{~nm}$ PSLA(1-4) surface}

SEM images of the composite families were captured and the interparticle distances between AuNPs on the surface was measured manually using Image software. At least 50 AuNPAuNP distances were measured for each composite family and the value of the closest particle-particle interacting distance was taken for each composite sample. For lowly occupied composites, any AuNPs outside the interacting distance $(>1.5 D$, where $\mathrm{D}$ is the AuNP diameter) with another AuNP were given a value equal to the $1.5 D$.

\section{SERS measurements on 200 nm PSLA4 composites}

SERS measurements were taken using a Renishaw inVia Raman microscope (50x objective) fitted with an $830 \mathrm{~nm}$ laser (maximum output of $56 \mathrm{~mW}$ ) and a $1200 \mathrm{l} / \mathrm{mm}$ diffraction grating. The SERS as a function of AuNP size was measured by adding $150 \mu \mathrm{L}$ of each composite family ( $\left.6 \times 10^{-3} \%(\mathrm{w} / \mathrm{v}) \mathrm{PS}\right)$ to an $850 \mu \mathrm{L}$ solution of $4-\mathrm{MP}(1 \mathrm{mM}) .^{22,54}$ These solutions were agitated at $1000 \mathrm{rpm}$ and $25^{\circ} \mathrm{C}$ for $45 \mathrm{~min}$, before centrifugation washing (5000 rpm, $20 \mathrm{~min}$ ) to remove excess 4-MP. Samples were re-dispersed in $100 \mu \mathrm{L}$ of Millipore $\mathrm{H}_{2} \mathrm{O}$ and $40 \mu \mathrm{L}$ was dropped onto cleaned $\mathrm{CaF}_{2}$ plates and dried. Raman spectra were recorded from similarly dense areas of composite materials for all samples, with $10 \mathrm{~s}$ exposure time, 1 accumulation and $7.6 \mathrm{~mW}$ laser power at the sample. Grid measurements were taken over a $15 \mu \mathrm{m} \times 15 \mu \mathrm{m}$ area with 1.9 $\mu \mathrm{m}$ step sizes ( $n=\sim 50$ measurements).

\section{Optical Tweezer SERS measurements on $5.3 \mu \mathrm{m}$ PSLA4 Composites}

Samples were prepared as above with a conc. of $0.15 \%(w / v)$ PS. Measurements were carried out in the Central Laser Facility in the Rutherford Appleton Laboratory in the UK on a custombuilt system. ${ }^{55}$ The system allowed simultaneous optical trapping and Raman excitation of particles and was equipped with a $785 \mathrm{~nm}$ laser (Spectra-Physics Ti:Sapphire laser pumped at $532 \mathrm{~nm}$ by an $8 \mathrm{~W}$ Coherent Verdi laser) and used a x60 NA 1.2 water immersion lens on an inverted microscope. Trapped particles were visualised under filtered brightfield illumination and a motorised stage allowed manipulation of the sample cell to avoid collisions in solution (see ESI, Scheme S1). Sample cells consisted of $22 \mathrm{~mm}$ cylindrical glass wells with a quartz coverslip bottom to minimise the background weak fluorescence associated with glass. Typically, $600 \mu \mathrm{L}$ of sample was loaded into the well for individual composites to be trapped and measured. All samples were trapped with a measured laser power of $20 \mathrm{~mW}$ at the objective pupil. The spectrograph (1200 $\mathrm{g} / \mathrm{mm}$ ) was calibrated using toluene as a standard and was accurate to 2 relative $\mathrm{cm}^{-1}$. Each trapped particle was measured with a $0.2 \mathrm{~s}$ exposure for 25 measurements, with 25 separate particles measured.

\section{Results and Discussion}

\section{Functionalisation of PS-COOH beads}

The optical properties of AuNP composite materials are related to the NP separation at the surface. To prepare materials with differing AuNP-AuNP distances a base support was selected that (i) showed no inherent affinity for the AuNPs and (ii) could be easily functionalised to different degrees, with a ligand that had high affinity for AuNPs. $200 \mathrm{~nm}$ carboxylic acid modified PS beads (PS-COOH) were chosen as they have low affinity for negatively charged citrate stabilised AuNPs and their size allows routine spectroscopic characterisation. The lipoic acid based 


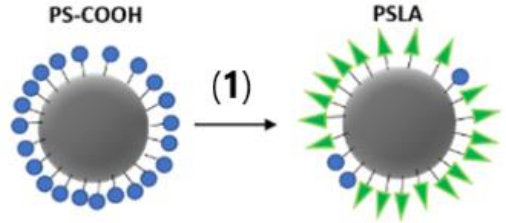

(a)

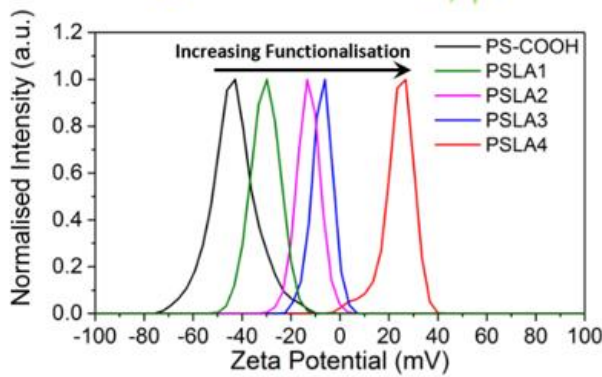

(b)

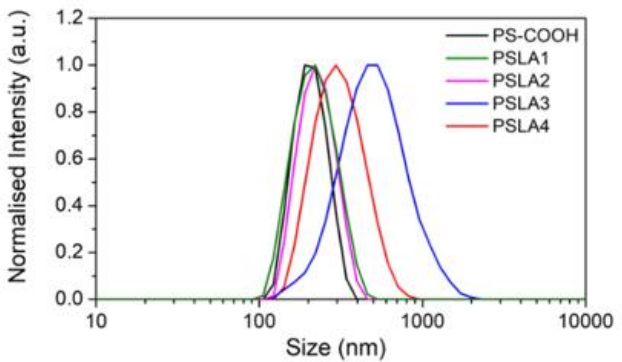

Fig. 1 (a) $\zeta$-potential and (b) DLS measurements of PS-COOH and lipoic amine modified PS (PSLA1-4) with various surface functionality. Measurements were taken in Millipore $\mathrm{H}_{2} \mathrm{O}$ at $\mathrm{pH} 7$.

molecule 1 was chosen as it has a high affinity for AuNPs. ${ }^{51,56-58}$ Carbodiimide chemistry was used to form the active ester at the PS-COOH surface, which was then reacted with the terminal amine of 1 to form a robust amide bond, (see Fig. S1, ESI). Control over the degree of surface functionalisation was achieved by changing (a) the molar ratio of surface carboxylic acid group to 1 and (b) the reaction time. The amount of surface $\mathrm{COOH}$ groups in each reaction was estimated using the surface occupancy provided by the manufacturer (see SI). PS beads functionalised with increasing amounts of 1 from PSLA1 to PSLA4 were prepared. The least functionalised PSLA1 family was prepared using a molar ratio of $\mathrm{COOH}: \mathrm{LA}$ of 1:0.33 with a reaction time of $5 \mathrm{~h}$. Increasing the reaction time to $16 \mathrm{~h}$ afforded PSLA2. The greatest functionalisation (PSLA4) was achieved using a molar ratio of 1:20 and a $16 \mathrm{~h}$ reaction time, see Table 1.

Table 1. Summary of reaction conditions, $\zeta$-potential changes and hydrodynamic radii of the different PSLA families

\begin{tabular}{ccccccc}
\hline System & COOH:LA & Rxn Time & \-potential & $\Delta \mathrm{mV}$ & $\begin{array}{c}\text { Hydrodynamic } \\
\text { Radius (nm) }\end{array}$ & PDI \\
\hline PS-COOH & - & - & $-47 \pm 6 \mathrm{mV}$ & - & $208 \pm 15$ & 0.061 \\
PSLA1 & $1: 0.33$ & $5 \mathrm{~h}$ & $-30 \pm 7 \mathrm{mV}$ & $+17 \mathrm{mV}$ & $221 \pm 31$ & 0.194 \\
PSLA2 & $1: 0.33$ & $16 \mathrm{~h}$ & $-13 \pm 3 \mathrm{mV}$ & $+34 \mathrm{mV}$ & $216 \pm 28$ & 0.159 \\
PSLA3 & $1: 0.75$ & $16 \mathrm{~h}$ & $-6 \pm 3 \mathrm{mV}$ & $+41 \mathrm{mV}$ & $516 \pm 230$ & 0.631 \\
PSLA4 & $1: 20$ & $16 \mathrm{~h}$ & $+27 \pm 6 \mathrm{mV}$ & $+73 \mathrm{mV}$ & $296 \pm 61$ & 0.286
\end{tabular}

It proved problematic to monitor the functionalisation with molecule 1 by FTIR due to the strong contribution of the vibrations arising from the $200 \mathrm{~nm}$ PS relative to the contribution of the surface groups. Therefore, the relative degree of surface functionalisation was monitored by measuring the $\zeta$-potential, which has previously been used to report on the surface functionalisation of PS particles. ${ }^{59}$ The $\zeta$ potential of the PS-COOH in water was found to be highly negative $(-47 \pm 6 \mathrm{mV})$ due to the presence of surface carboxylate groups. The negative surface charge was found to be reduced upon reaction with 1 and a clear trend was observed progressing from conditions chosen for low functionalisation, $-30 \pm 7 \mathrm{mV}$ (PSLA1) to high functionalisation, $+27 \pm 6 \mathrm{mV}$ (PSLA4), see Fig. 1a, Table 1.

DLS measurements of the PS-COOH support showed a hydrodynamic radius of $208 \pm 15 \mathrm{~nm}$, which was found to increase slightly for PSLA1 and PSLA2, with no signs of aggregation or agglomeration, see Table 1. However, the PSLA3 beads showed a significant increase in the hydrodynamic radius of $516 \pm 230 \mathrm{~nm}$ and a polydispersity index (PDI) of 0.631, which suggested some aggregation. This observed behaviour is taken to reflect the low value of $\zeta$-potential of the PSLA3. The beads were successfully re-dispersed using sonication; however, they did not remain dispersed in solution for more than $30 \mathrm{~s}$ without agitation. The properties of the systems are summarised in Table 1.

\section{Assembly of AuNP composites}

Next, the formation of composites using PSLA(1-4) and AuNPs was investigated. Five sizes of citrate stabilised AuNPs between $13 \mathrm{~nm}$ and $30 \mathrm{~nm}$ were synthesised using the seed growth mechanism developed by Bastus et al.. ${ }^{53}$ The AuNPs were fully characterised by UV-vis, AAS and TEM (see Fig. S2 \& Table S2, ESI). Analysis of TEM images revealed the families to have size distribution of $13 \pm 2 \mathrm{~nm}, 17 \pm 2 \mathrm{~nm}, 22 \pm 3 \mathrm{~nm}, 26 \pm 3 \mathrm{~nm}$ and $30 \pm 3 \mathrm{~nm}$. The position of the SPR band was found to range from $517 \mathrm{~nm}$ to $527 \mathrm{~nm}$ and had an average $\zeta$-potential of -40 $\pm 3 \mathrm{mV}$. Composite particles were prepared by adding aqueous solutions of the different sized AuNPs to aqueous solutions of the PSLA1-4 beads. These samples were shaken for $60 \mathrm{~min}$ and washed by mild centrifugation to remove free AuNPs. In this way twenty composite samples were prepared. The composite samples were dispersed readily in Millipore $\mathrm{H}_{2} \mathrm{O}$ via sonication and remained stable to sedimentation.
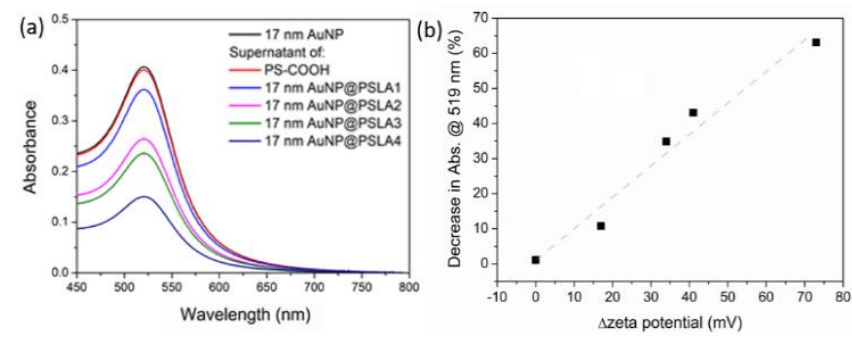

Fig. 2 (a) Visible absorption spectra of $17 \mathrm{~nm}$ AuNP control as well as supernatants after composite formation with PSLA1-4. (b) Graph showing a linear decrease in absorbance as a function of $\zeta$-potential shift after functionalisation for $17 \mathrm{~nm}$ AuNPs. 
(a)
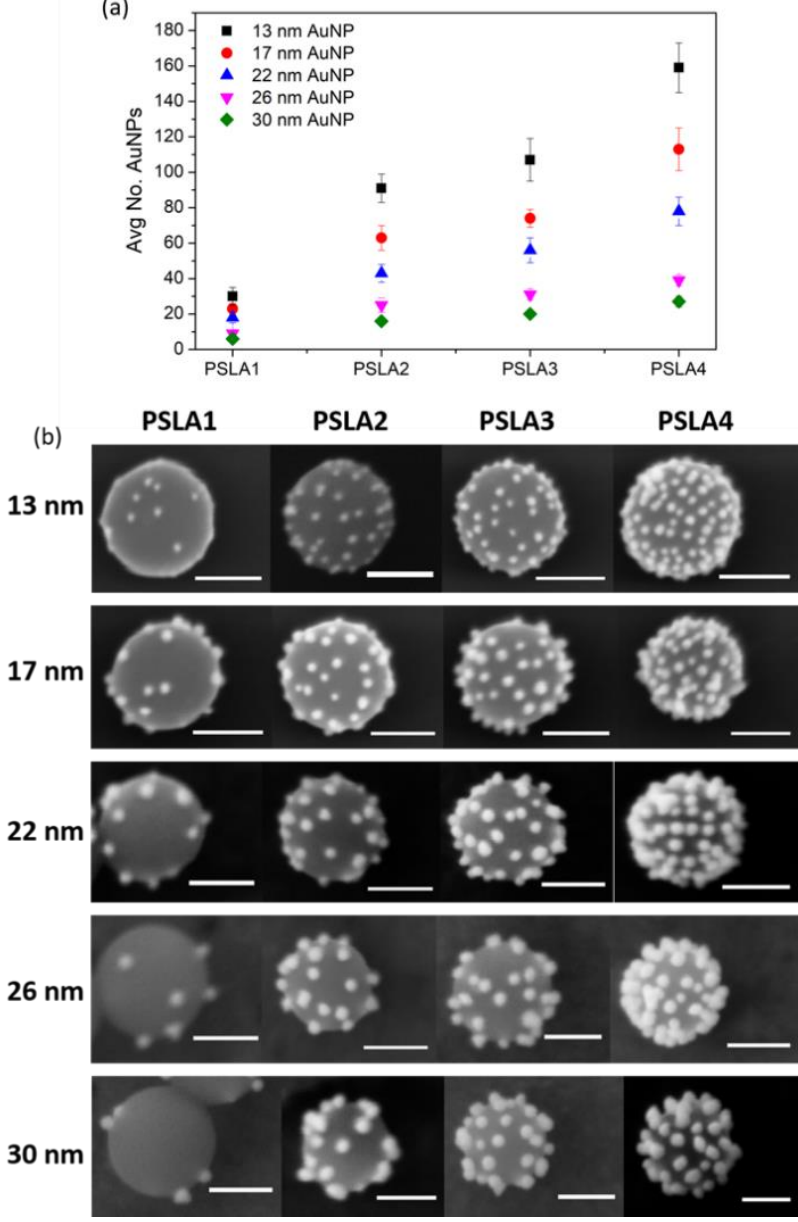

Fig. 3 (a) Graph showing No. AuNPs immobilised on the surface of the PSLA1-4 beads for each family (Taken from the avg. determined by UV-vis and AAS) (b) SEM images of different composite families. Left legend indicates AuNP size and top legend indicates PSLA family (scale bar $=100 \mathrm{~nm}$ for all images).

The recovered supernatant was analysed by UV-visible absorption spectroscopy. As expected, no loss in solution [AuNP] was detected for AuNPs added to PS-COOH. In contrast, analysis of the supernatant recovered from PSLA1-4 showed a decrease in the concentration of free AuNPs from sample PSLA1 to PSLA4. The trend observed for the $17 \mathrm{~nm}$ AuNPs is shown in Fig. 2 (a), for the other sizes see ESI Fig. S3. Notably, the decrease of the SPR absorbance at $519 \mathrm{~nm}$ for the supernatant of $17 \mathrm{~nm}$ AuNPs was found to be directly proportional to the change in $\zeta$-potential observed upon functionalisation of the PSCOOH with 1, see Fig. 2(b).

UV-visible spectroscopy and AAS measurements of the supernatant were used to determine the surface loading, which was found to increase proportionally for composites from PSLA1 to PSLA4 for all NP sizes, see Fig. 3 (a). For the $17 \mathrm{~nm}$ family the estimated number of AuNPs per PS bead, as determined by both techniques, was on average $24 \pm 4$ (PSLA1) and $112 \pm 12$ (PSLA4), Table 2. These NP loadings represent an average PS surface area coverage of $4.1 \%$ (PSLA1) and $20 \%$ (PSLA4). The value for the maximum loaded $17 \mathbf{~ n m}$ AuNP@PSLA4 is typical for electrostatically stabilised AuNPs (<30\%) due to Coulomb repulsions ${ }^{19,43,60}$ and is in agreement

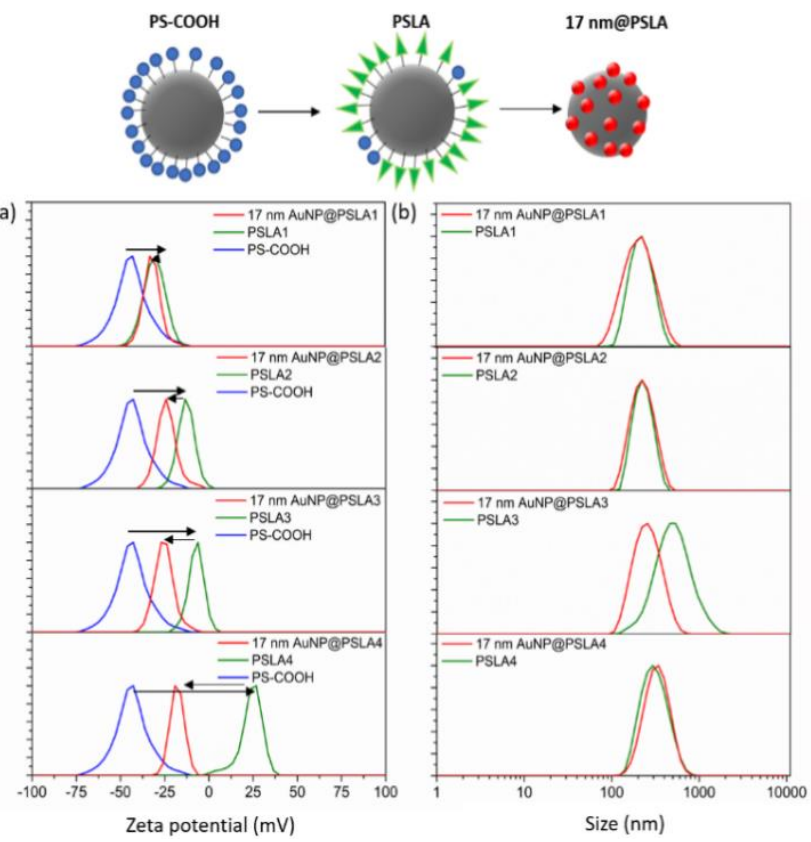

Fig. 4 Characterisation of composite formation (a) $\zeta$-potential and (b) DLS measurements of $\mathbf{1 7}$ nm AuNP@PSLA1-4 composite materials taken in Millipore $\mathrm{H}_{2} \mathrm{O}$ at $\mathrm{pH} 7$.

with our previously reported values for AuNPs immobilised onto amine-modified PS beads. ${ }^{14}$ Similar degrees of coverage (3-23 \%) were determined for the other NP sizes, see ESI, Table S3.

SEM characterisation of the composite materials formed for the different AuNP sizes clearly revealed the incremental change in the population of surface immobilised AuNPs, see Fig. 3 (b). Importantly, no free AuNPs were observed in the background of the SEM images, indicating that centrifugal washing removed all unbound AuNPs from solution. The change in the PS bead surface charge due to composite formation was monitored by measuring the $\zeta$-potential of the samples. The results for the $\mathbf{1 7} \mathbf{~ n m ~ A u N P @ P S L A ~ c o m p o s i t e ~ f a m i l y ~ a r e ~ s h o w n ~}$ in Fig. 4(a), which shows the sequential $\zeta$-potential recorded upon (i) surface functionalisation with 1 and (ii) the subsequent immobilisation of AuNPs. A greater negative shift in $\zeta$-potential was observed for AuNP composites PSLA1 to PSLA4, which is indicative of increased binding of the negatively charged AuNPs. This trend is clearly shown for all the composites prepared (see ESI, Fig. S4). DLS measurements reveal a single peak for all composites, with the average size ranging from $238 \pm 47 \mathrm{~nm}$ to $310 \pm 52$ nm, Fig. 4(b) and ESI, Fig. S5-S8. No evidence for the presence of free AuNPs in solution or aggregation of composites is observed, which indicates that no cross-linking of composite particles though interaction of free anchor groups occurred. Notably, the AuNP@PSLA3 composites demonstrate increased colloidal stability compared to the bare PSLA3 beads for all sizes, reflecting the shift in the $\zeta$-potential towards a more negative value.

\section{Optical properties of AuNP composites}

As the AuNP loading increased the colour of the composites dispersed in aqueous solution was found to change pink/red to purple. In all cases the visible spectrum showed the presence of 

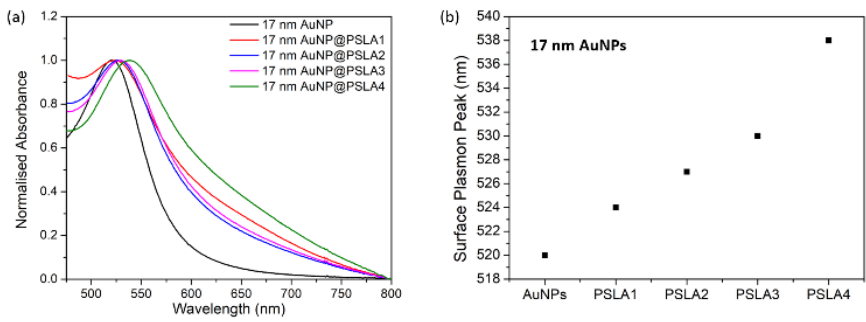

Fig. 5 (a) Visible absorption spectra of $17 \mathrm{~nm}$ AuNPs and composite families $\mathbf{1 7} \mathbf{n m}$ AuNPs@PSLA1-4 recorded in Millipore $\mathrm{H}_{2} \mathrm{O}$. (b) Graph showing surface plasmon peak position for each of the formed composite particles.

a SPR band characteristic of the presence of AuNPs, which was found to be red shifted from the SPR of the AuNP in solution, see Fig. 5(a), ESI S9. Furthermore, the SPR red-shift increased with increasing AuNP surface coverage (PSLA1 to PSLA4). This change arises due to the AuNP interaction with (i) the PS surface and (ii) nearby AuNPs. The normalised spectra recorded for the $17 \mathrm{~nm}$ AuNP family and the corresponding trend in the shift in the SPR is illustrated in Fig. $\mathbf{5 b}$. The spectra also reveal greater absorption at wavelengths $>600 \mathrm{~nm}$ which is characteristic of AuNP coupling. ${ }^{61}$ The different loadings resulted in changes in the SPR band position of up to $25 \mathrm{~nm}$ (see Table S5, ESI). This demonstrates the tunability of the AuNPs@PSLA systems. Absorption at wavelengths $>650 \mathrm{~nm}$ was observed for two families, 26 nm AuNP@PSLA4 and 30 nm AuNPs@PSLA3 and may be due to some aggregation occurring for these samples.

In light of the trend observed in the SPR shift with AuNP loading the optical properties of the AuNP families were examined using the universal ruler equation (1): 35

$$
\Delta \lambda / \lambda_{0}=\operatorname{axp}((-s / D) / t)
$$

Equation 1 relates changes in the optical properties to the AuNP separation. Specifically, the expression equates the fractional surface plasmonic shift $\left(\Delta \lambda / \lambda_{0}\right.$, where $\Delta \lambda$ is the shift in surface plasmon peak position, $\lambda_{0}$ is the original surface plasmon peak position of the isolated NP) with the relative nanoparticle separation $(s / D), s$ is the interparticle separation, $D$ the nanoparticle diameter. The amplitude of the shift, given by the pre-exponential factor $a$, depends on the shape and size of the NPs and on the dielectric environment and typically has values ranging between 0.08 to 0.18 for spherical AuNPs. ${ }^{33}$ The distance decay constant $(t)$ has been observed to be independent of the size of spherical NPs and the optical environment, and a value of 0.2 is typically taken. ${ }^{33}$ In order to

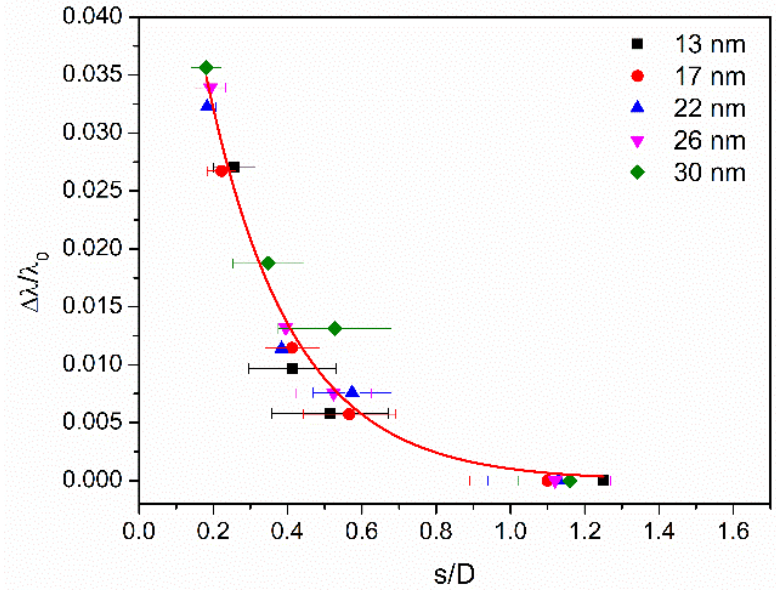

Fig. 6. Fractional surface plasmon shift $\left(\Delta \lambda / \lambda_{\text {OPSLA }}\right)$ plotted as a function of relative interparticle distance (s/D) for all composites.

apply equation (1), the NP diameter was determined from TEM measurements of at least 100 AuNPs. While the AuNP-AuNP distance on the surface was determined via SEM analysis, (see Table S6 \& S7, ESI).

As noted above, an AuNP-AuNP separation $>1.5 D$ results in a negligible effect on the SPR band. ${ }^{33}$ Analysis of the SEM images recorded for 17nm AuNP@PLSA1 revealed $85 \%$ of the AuNPs to be outside this range (see ESI, Fig. S10-S11), with an average NP separation of $34.3 \pm 12.3 \mathrm{~nm}$. However, when this population's separation was normalised to $1.5 D$ the average NP separation for the sample was found to be $18.7 \pm 2.4 \mathrm{~nm}$. In contrast, all $17 \mathrm{~nm}$ AuNPs were within coupling distance for the fully loaded system (PSLA4) where the separation was found to be $3.8 \pm 0.7 \mathrm{~nm}$. Due to the difference in relative refractive index between water (1.33) and polystyrene (1.55-1.6), the $\lambda_{\text {SPR }}$ of discrete AuNPs at the PSLA bead surface is expected to be different to that for discrete AuNPs in solution. ${ }^{14,62}$ However, given the majority of the NP population for the PSLA1 composites were found to be separated by distances greater than $1.5 \mathrm{D}$, the $\lambda_{0(P S L A)}$ was taken to be equal to the value recorded for the least occupied PSLA1 composite SPR( $\left.\lambda_{\text {SPR(PSLA1) }}\right)$ for each NP family.

The plot of $\Delta \lambda / \lambda_{0 \text { (PLSA) }}$ against average relative interparticle distance (S/D) at the PS bead surface was fitted to a monoexponential function for all NP sizes, see Fig 6 and Fig. S12. Fitting of the data revealed the optical properties of the composites to be well described by a universal ruler equation. The optimised global fitting parameters of $a(0.076 \pm 0.006)$ and $t(0.233 \pm 0.023)$ are similar to values previously determined

Table 2. Summary of $\zeta$-potential changes pre- and post-composite formation and the loading of AuNPs on the surface of the composite materials formed using $17 \mathrm{~nm}$ AuNPs

\begin{tabular}{|c|c|c|c|c|c|c|c|}
\hline \multirow{3}{*}{$\begin{array}{c}\text { Family } \\
17 \text { nm AuNP@ }\end{array}$} & \multirow{3}{*}{$\begin{array}{c}\text { Unmodified PS Bead } \\
\zeta \text {-potential }\end{array}$} & \multirow{3}{*}{$\begin{array}{c}\text { Composite } \\
\text { Material } \\
\text { ל-potential }\end{array}$} & \multirow{3}{*}{$\Delta \zeta$-potential } & \multicolumn{2}{|c|}{ UV-vis Loading } & \multicolumn{2}{|c|}{ AAS Loading } \\
\hline & & & & No. AuNPs & $\%$ PS SA & No. AuNPs & $\%$ PS SA \\
\hline & & & & & Covered & & Covered \\
\hline PSLA1 & $-30 \pm 3 m V$ & $-33 \pm 2 m V$ & $-3 m V$ & $20 \pm 3$ & $3.6 \%$ & $27 \pm 4$ & $4.7 \%$ \\
\hline PSLA2 & $-13 \pm 3 m V$ & $-26 \pm 3 m V$ & $-13 m V$ & $63 \pm 7$ & $11.3 \%$ & $67 \pm 7$ & $11.3 \%$ \\
\hline PSLA3 & $-6 \pm 2 m V$ & $-27 \pm 3 m V$ & $-21 \mathrm{mV}$ & $76 \pm 5$ & $13.7 \%$ & $72 \pm 5$ & $13.0 \%$ \\
\hline PSLA4 & $+27 \pm 3 \mathrm{mV}$ & $-19 \pm 2 m V$ & $-46 m V$ & $114 \pm 12$ & $20.6 \%$ & $110 \pm 12$ & $19.9 \%$ \\
\hline
\end{tabular}


experimentally and using discrete dipole approximation (DDA) calculations for coupled spherical AuNP systems. ${ }^{33}$ The slightly lower $a$ value obtained compared to reported values may be due to the presence of AuNPs on the surface of the PSLA beads experiencing surface plasmon coupling with multiple particles, as opposed to a single AuNP, as is the case with dimers. The controlled loading of the different sized AuNPs allows access to tunable SPR between 518 and $552 \mathrm{~nm}$. In the case of the $13 \mathrm{~nm}$ The SPR ranges from $518 \mathrm{~nm}$ to $532 \mathrm{~nm}$ and for $30 \mathrm{~nm}$ AuNP from $533 \mathrm{~nm}$ to $552 \mathrm{~nm}$.

\section{Size dependent SERS response of AuNP@PS composites} Bulk measurement of SERS activity

The SERS activity of the fully loaded AuNP@PSLA composites was tested using 4-mercaptophenol (4-MP) as Raman reporter molecule, and selected because it is known to bind strongly to AuNPs via the thiol moiety. ${ }^{22,63}$ In the first approach bulk measurements were performed on the composite material deposited on a $\mathrm{CaF}_{2}$ using a Raman microscope using $830 \mathrm{~nm}$ excitation which is non-resonant with the composite particles, see Fig. 5(a). The $830 \mathrm{~nm}$ non-resonant laser source was chosen as it allows direct comparison of the different composites under conditions which are relevant for biological applications ${ }^{64}$ and also avoids any possible annealing or heating effects on the SERS signal. 65 The Raman spectrum recorded for 4-MP deposited on the $\mathrm{CaF}_{2}$ plate shows a small peak centred at 1075 $\mathrm{cm}^{-1}$, characteristic of the 4-MP ring breathing mode and a larger peak centred at $318 \mathrm{~cm}^{-1}$ due to $\mathrm{CaF}_{2}$ (see ESI, Fig. S13)

(a)
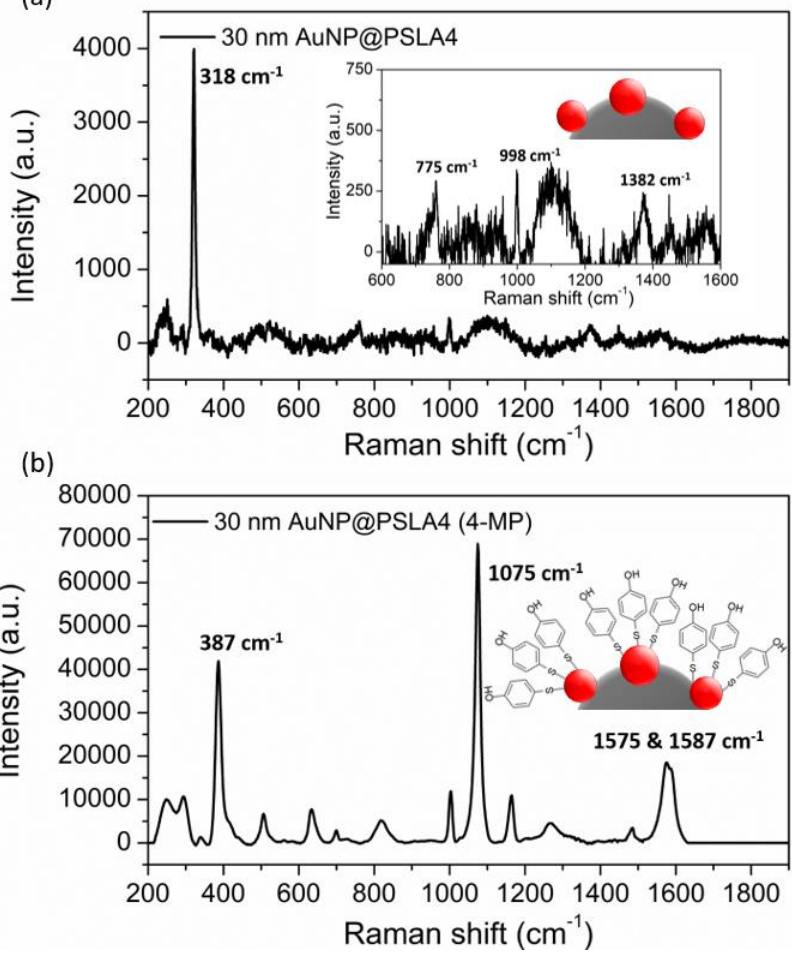

Fig. 7. Raman spectra recorded for (a) 30 nm AuNP@PSLA4 composites and (b) 30 nm AuNP@PSLA4 composites incubated with 4-MP all deposited on $\mathrm{CaF}_{2}$ plates. Al samples were measured with a $10 \mathrm{~s}$ exposure time with a $\lambda_{\text {ex }}=830 \mathrm{~nm}$ with 1 accumulation and a laser power of $7.6 \mathrm{~mW}$. ( ${ }^{*}$ denotes peak associated with $\mathrm{CaF}_{2}$ substrate).
The Raman spectra obtained for the 30 nm AuNP@PSLA4 system with, and without 4-MP incubation are illustrated in Fig. 7. The Raman spectrum of $30 \mathrm{~nm}$ AuNP@PSLA4 composite material is dominated by the CaF2 substrate signal dominates with several additional low intensity peaks between $750 \mathrm{~cm}^{-1}$ and $1400 \mathrm{~cm}^{-1}$, see Fig. 7 (a). The peaks at $775 \mathrm{~cm}^{-1}$ and 1382 $\mathrm{cm}^{-1}$ are attributed to Raman scattering from the citrate ions stabilising the AuNPs, ${ }^{66}$ while the peak at $998 \mathrm{~cm}^{-1}$ is assigned to Raman scattering contributions from the PS bead. ${ }^{67}$

In contrast, Raman measurements of $\mathbf{3 0}$ nm AuNP@PSLA4 composite particles incubated with 4-MP gave a highly structured spectrum with several well-resolved peaks characteristic of 4-MP, see Fig. 7 (b), Table S7, ESI). ${ }^{68}$ The most prominent peak was centred at $1075 \mathrm{~cm}^{-1}$, the ring breathing mode of the aromatic ring, and is significantly enhanced ( 70000 intensity units) compared to the control measurements on 4-MP ( 100 intensity units). Importantly, the peak associated with PS (998 $\mathrm{cm}^{-1}$ ) is no longer prevalent, but a peak at $1002 \mathrm{~cm}^{-1}$ is observed associated with 4-MP. The SERS activity of AuNPs has been shown to be related to the size of the AuNPs, ${ }^{69-71}$ and measurements demonstrate how activity increases in the range 10 - $60 \mathrm{~nm} \cdot{ }^{69,71}$ In this current study the relationship is examined for composite materials by recording the Raman spectra for the different fully loaded composite materials following incubation with 4-MP and washing. All AuNP@PSLA4 systems displayed Raman bands characteristic of 4-MP, (Fig 8. (a) \& (b)). Comparison of the signal intensity at $1075 \mathrm{~cm}-1$ gave a non-linear increase in the Raman scattering with increasing diameter from $\sim 350$ and 70000 intensity units for the $13 \mathbf{~ n m}$ AuNP@PSLA4 and $30 \mathrm{~nm}$ AuNP@PSLA4 composites respectively, see Fig. 8(c). A key challenge for SERS technology to become a routine analytical method is ensuring reproducibility of signal enhancements factors from substrate to substrate.72 The reproducible nature of the SERS measurements was determined by comparing data from two separately made samples (30 nm AuNP@PSLA4) with approximately about $15 \mu \mathrm{m} \times 15 \mu \mathrm{m}$ area. These measurements yielded an average Raman intensity for the $1075 \mathrm{~cm}-1$ mode of 4-MP of $68914 \pm 14322$. We ascribe this significant error to the observed $20 \%$ deviation in the interparticle distances recorded for the PSLA4 samples and the inherent differences in the PS size.

This trend in the Raman signal and NP size persisted when the Raman intensity was adjusted to reflect the concentration of 4-MP on the surface, as estimated for each composite by assuming monolayer coverage of the AuNP with a molecular footprint of $0.33 \mathrm{~nm}^{2}, 22$ (see Table S8), see Fig. 8 (d). A nonlinear increase in SERS intensity as a function of AuNP of a similar size range has been previously reported. ${ }^{69,70}$ The improved response for larger NPs is attributed to an increase of the active hot-spot size, ${ }^{25}$ due to (i) a decrease in the relative interparticle separation and (ii) an increase in the spectral shift of the SPR band as a function of increasing AuNP diameter. It is expected that these effects contribute to the improved performance of the $\mathbf{3 0} \mathbf{n m}$ AuNP@PSLA4 composite. 
(a)
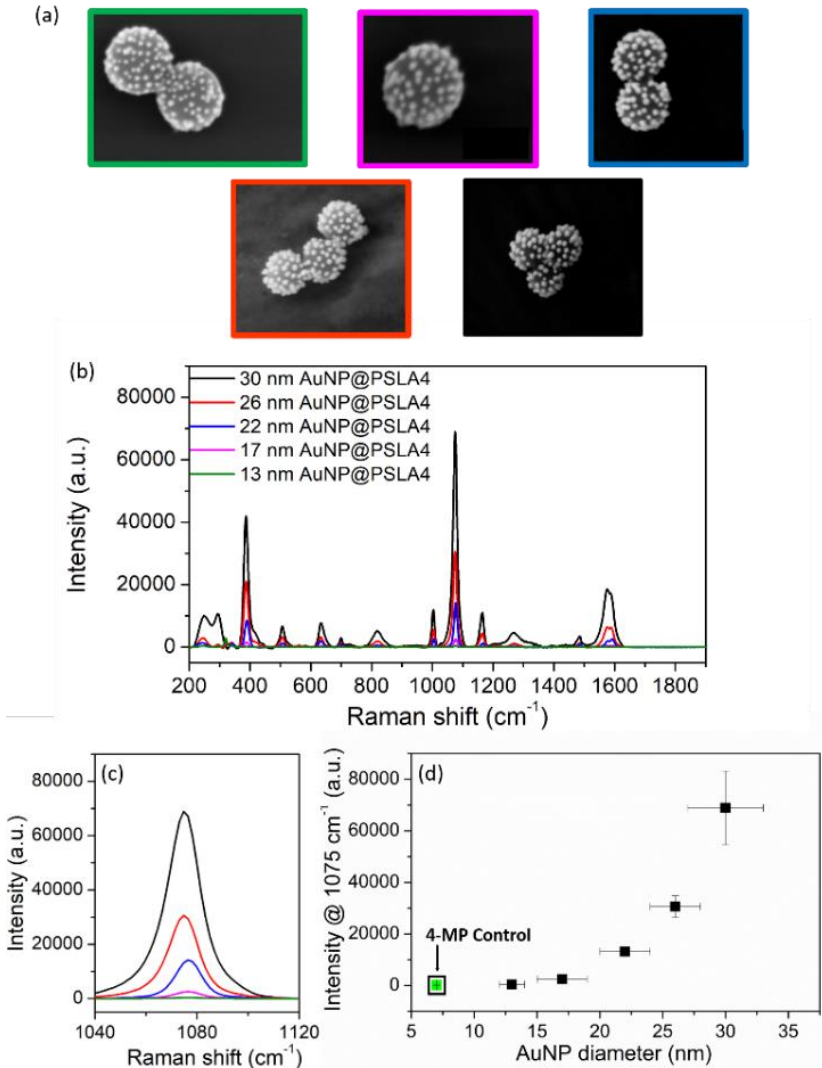

Fig. 8 (a) SEM images of $13 \mathrm{~nm}$ AuNP, $17 \mathrm{~nm}$ AuNP, $22 \mathrm{~nm}$ AuNP, $26 \mathrm{~nm}$ AuNP, and 30 nm @PSLA4 composites used for SERS study. (b) Raman spectra generated from 4MP labelled AuNP@PSLA4 composites. (c) Raman spectra between $1040 \mathrm{~cm}^{-1}$ and $1120 \mathrm{~cm}^{-1}$ for all composites measured. (d) Graph showing the intensity at $1075 \mathrm{~cm}^{-1}$ normalised for the relative number of 4-MP molecules immobilised as a function of AuNP diameter. All samples were measured upon $\lambda_{\text {ex }}=830 \mathrm{~nm}$, for a $10 \mathrm{~s}$ exposure time with 1 accumulation and a laser power of $7.6 \mathrm{~mW}$ in the ca. $2 \mu \mathrm{m}$ focused spot.

\section{Single Particle measurement of SERS activity}

The use of Raman optical tweezers is particularly attractive for the current study, as the bulk SERS response may also include contributions of hot spots arising due to multi-layer, 3D arrays of composite materials and plasmonic interactions between composite particles adjacent to each other. To probe the SERS activity of individual composite particles in solution, larger $5.3 \mu \mathrm{m}$ PS-COOH scaffold PS bead were fully functionalised with molecule 1 to prepare $5.3 \mu \mathrm{m}$ PSLA4 beads. Using these scaffolds, composite materials were then formed with 13-30 nm AuNPs (see ESI, Fig. S14). The surface coverage of the AuNPs was determined using UV-vis and AAS and to be $17-22 \%$, which was comparable to composites formed using $200 \mathrm{~nm}$ PSLA4 beads (see ESI, Table S9). Single particle SERS measurements were performed using a non-resonant $785 \mathrm{~nm}$ laser for trapping and Raman measurement. Each measurement was performed on 25 separate individual composite particles. The Raman spectrum of optically trapped $5.3 \mu \mathrm{m}$ PSLA4 was found to be in good agreement with that previously reported for optically trapped PS beads and is dominated by the breathing mode of the aromatic ring of the PS at $998 \mathrm{~cm}^{-1} .73$

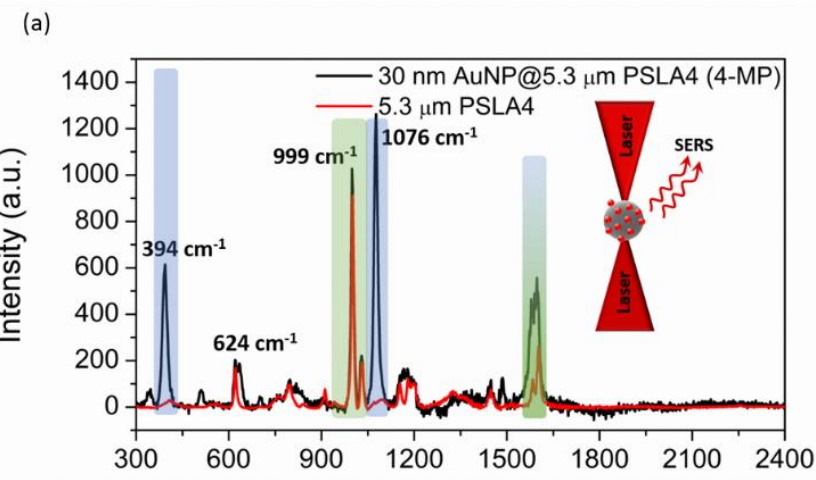

(b)

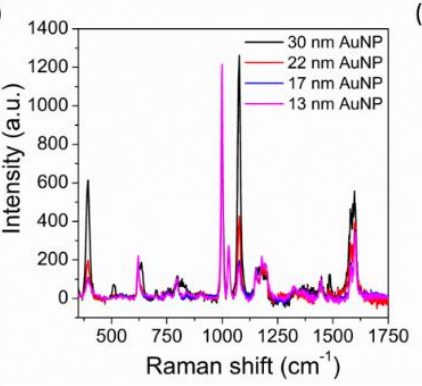

(c)

(d)

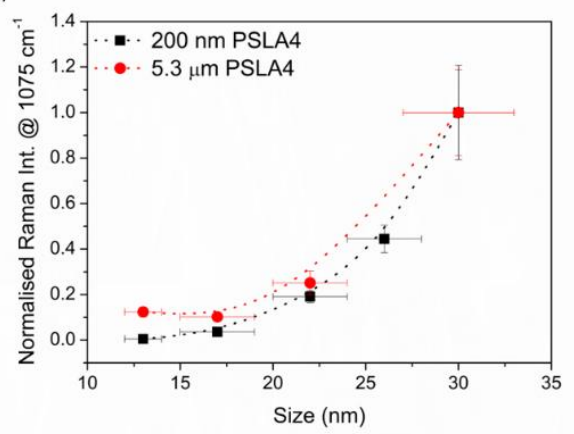

Fig 9. (a) Raman spectra generated from AuNP@5.3 um PSLA4 and 30 nm AuNP@5.3 $\mu \mathrm{m}$ PSLA4 incubated with 4-MP. Blue shade indicates regions with strong peaks associated with 4-MP whilst green shade indicates regions with strong peaks associated with PS (b) Raman spectra generated from 4-MP labelled AuNP@5.3 $\mu \mathrm{m}$ PSLA4 composite materials. (c) Raman spectra between $1040 \mathrm{~cm}^{-1}$ and $1150 \mathrm{~cm}^{-1}$ for all composites measured. Spectra were recorded in Millipore $\mathrm{H}_{2} \mathrm{O}$ using the same laser source $(\lambda e x=785 \mathrm{~nm}$ ) for both trapping and SERS excitation with a laser power of 20 $\mathrm{mW}$ at the objective, a $0.2 \mathrm{~s}$ exposure time and 1 accumulation. (d) Comparison of the normalised Raman intensity at $1075 \mathrm{~cm}^{-1}$ for both composite formed using $13-30$ $\mathrm{nm}$ AuNPs on $200 \mathrm{~nm}$ PSLA and $5.3 \mu \mathrm{m}$ PSLA (PSLA4) beads.

Notably, there were no contributions between $1050 \mathrm{~cm}^{-1}$ and $1120 \mathrm{~cm}^{-1}$, where the most intense band for 4-MP lies (see Fig 9a).

Furthermore, the Raman spectrum recorded for an optically trapped 5.3 $\mu \mathrm{m}$ PSLA4 bead incubated with 4-MP showed no contribution of 4-MP in the spectrum (see ESI, Fig. S15). While the presence of a reflective metal surface is expected to impede the trapping, it was possible to readily trap the AuNP@5.3 $\mu \mathrm{m}$ PSLA4 composite particles in this study. Raman spectrum of $\mathbf{3 0}$ nm AuNP@PSLA4 particles incubated in 4-MP reveal peaks associated 4-MP at $394 \mathrm{~cm}^{-1}$ and $1076 \mathrm{~cm}^{-1}$ (see Fig. 9a), indicative of a SERS response from the 4-MP labelled composite material. The intensity of the PS band at $999 \mathrm{~cm}^{-1}$ is found to be greater in the trapped spectrum due to the greater relative contribution of the larger PS scaffold. The difference in the 
relative peak intensities observed for the bulk and tweezers spectra of 4-MP, following normalisation of spectral intensity, are ascribed to the use of different laser excitation wavelengths, 830 and $785 \mathrm{~nm}$ respectively. Similar differences in the Raman spectral profile of 4-MP were previously observed using laser wavelengths from 488 to $785 \mathrm{~nm}$ and were attributed to the change in laser wavelengths permits a relative tuning between the electromagnetic and/or chemical enhancements contributing to the SERS signals and a charge transfer mode of 4-MP. ${ }^{74}$

The SERS response of optically trapped AuNP $5.3 \mu \mathrm{m}$ PSLA4 composite particles, for AuNP sizes 13-30 nm was found to increase with size, with a similar trend observed compared to the AuNP@200 nm PSLA composites (see Fig. 9(b-c)) with similar adjustments made for conc. of 4-MP on the surface (see ESI, Table 10). These results demonstrate the ability to perform single composite Raman measurements with minute amounts of material, with an average of $0.367 \pm 0.037 \mathrm{fmol}$ of 4-MP immobilised onto the surface of the composite materials. Importantly, the use of the optical tweezer method allows the behaviour of the discrete composite to be assessed and suggests that the behaviour in the bulk is dominated by the discrete properties and not due to interactions between deposited composites.

\section{Conclusions}

Tuning the optical properties of nanomaterials by controlled NP assembly is of interest for several applications including SERS. In this work we have achieved controlled surface functionalisation of $200 \mathrm{~nm}$ PS beads by a lipoic acid derivative with known affinity for AuNPs. Defined diameter AuNPs were used to prepare colloidally stable AuNP@PS composite materials with different AuNP loadings, whose coverage was indicated by $\zeta$-potential measurements and confirmed by, UVvis, AAS and SEM. The composite materials displayed discrete and tunable surface plasmon resonance profiles between 518 and $552 \mathrm{~nm}$. The coupling behaviour was modelled to the surface plasmon ruler equation, revealing a mono-exponential decrease in the shift of the SPR wavelength maximum as a function of increased AuNP interparticle distance, consistent with AuNP dimer studies. ${ }^{31,33}$ The modest changes in the SPR observed for the composites are in line with previous observations for comparable separations of spherical AuNPs. Future work will investigate the plasmonic properties of anisotropic metal nanoparticles, which exhibit significantly greater shifts in the SPR wavelength and enhancements due to the enhanced SPR located at protrusions with high curvature, which give rise to the highest polarisability. ${ }^{75}$

SERS measurements demonstrated large enhancements of Raman intensity for the test molecule 4-MP, with a strong size dependence on the SERS response of the AUNPs recorded by both bulk and optical tweezer methods. There is significant interest in the development of cellular SERS probes and plasmonics, ${ }^{76-78}$ including point of care applications. ${ }^{79}$ This study extends our previously reported work examining the ability of cells to internalise AuNP@PS composites using dark field microscopy ${ }^{14}$ and further work will consider the SERS activity of individual composite particles as potential cellular probes. We believe that the reproducible preparation of the composites together with the tunability of the SPR band position and the stability indicate potential for their use for a number of applications such as radiosensitisation, payload delivery and cellular imaging.

\section{Conflicts of interest}

There are no conflicts to declare.

\section{Acknowledgements}

This work was supported by the Irish Research Council (GOIP/2013/1110, SAB) and UCD. The Raman experiments were performed through the award of access to the Central Laser Facility, STFC, App17230098. We are grateful to Prof. Kenneth Dawson and CBNI in UCD for access to DLS instrumentation. SEM and TEM imaging were carried out at the Advanced Microscopy Laboratory (AML) at the AMBER centre, CRANN institute (https://www.crann.tcd.ie/Facilities/ AdvancedMicroscopy-Laboratory.aspx), Trinity College Dublin, Ireland.

\section{References}

1 E. Roduner, Chem. Soc. Rev., 2006, 35, 583-592.

2 P. K. Jain, X. Huang, I. H. El-Sayed and M. A. El-Sayed, Acc. Chem. Res., 2008, 41, 1578-1586.

3 S. Link and M. a. El-Sayed, J. Phys. Chem. B, 1999, 103, 42124217.

4 C. Noguez, J. Phys. Chem. C, 2007, 111, 3606-3619.

5 M. R. Gartia, A. Hsiao, A. Pokhriyal, S. Seo, G. Kulsharova, B. T. Cunningham, T. C. Bond and G. L. Liu, Adv. Opt. Mater., 2013, 1, 68-76.

6 S. Underwood and P. Mulvaney, Langmuir, 1994, 10, 34273430.

7 M. M. Schmidt, S. Wu, Z. Cui, N. T. Nguyen, M. Faulkner and B. R. Saunders, Phys. Chem. Chem. Phys., 2017, 19, 5102-5112.

8 W. Hou and S. B. Cronin, Adv. Funct. Mater., 2013, 23, 16121619.

9 M. Brust and G. J. Gordillo, J. Am. Chem. Soc., 2012, 134, 33183321.

10 K.-S. Lee and M. a El-Sayed, J. Phys. Chem. B, 2006, 110, 1922019225.

11 A. Cifuentes-Rius, H. De Puig, J. C. Y. Kah, S. Borros and K. Hamad-Schifferli, ACS Nano, 2013, 7, 10066-10074.

12 K. T. Butterworth, S. J. McMahon, F. J. Currell and K. M. Prise, Nanoscale, 2012, 4, 4830-4838.

13 Z. Lu, J. Goebl, J. Ge and Y. Yin, J. Mater. Chem., 2009, 19, 45974602.

14 S. A. Belhout, J. Y. Kim, D. T. Hinds, N. J. Owen, J. A. Coulter and S. J. Quinn, Chem. Commun., 2016, 52, 14388-14391.

15 G. Agrawal, M. P. Schürings, P. van Rijn and A. Pich, J. Mater. Chem. A, 2013, 1, 13244-13251.

16 M. Li and G. Chen, Nanoscale, 2013, 5, 11919-11927.

17 Y. Liu, M. Li and G. Chen, J. Mater. Chem. A, 2013, 1, 930-937.

18 S. L. Westcott, S. J. Oldenburg, T. R. Lee and N. J. Halas, Chem. 
Phys. Lett., 1999, 300, 651-655.

19 S. L. Westcott, S. J. Oldenburg, T. R. Lee and N. J. Halas, Langmuir, 1998, 14, 5396-5401.

20 R. Kumar, E. Gravel, A. Hagège, H. Li, D. V. Jawale, D. Verma, I. N. N. Namboothiri and E. Doris, Nanoscale, 2013, 5, 6491-6497.

21 Y. Shi, H. Dai, Y. Sun, J. Hu, P. Ni and Z. Li, Analyst, 2013, 138, 7152-7156.

22 A. B. Serrano-Montes, J. Langer, M. Henriksen-Lacey, D. Jimenez De Aberasturi, D. M. Solís, J. M. Taboada, F. Obelleiro, K. Sentosun, S. Bals, A. Bekdemir, F. Stellacci and L. M. Liz-Marzán, J. Phys. Chem. C, 2016, 120, 20860-20868.

23 D. Radziuk and H. Moehwald, Phys. Chem. Chem. Phys., 2015, 17, 21072-21093.

24 L. Guerrini and D. Graham, Chem. Soc. Rev., 2012, 41, 70857107.

25 K. Kim, D. Shin, K. L. Kim and K. S. Shin, Phys Chem Chem Phys, 2010, 12, 3747-3752.

26 L. Guerrini, F. McKenzie, A. W. Wark, K. Faulds and D. Graham, Chem. Sci., 2012, 3, 2262-2269.

27 L. Velleman, L. Scarabelli, D. Sikdar, A. A. Kornyshev, L. M. LizMarzán and J. B. Edel, Faraday Discuss., 2017, 205, 67-83.

28 C. K. K. Choi, Y. T. E. Chiu, X. Zhuo, Y. Liu, C. Y. Pak, X. Liu, Y. L. S. Tse, J. Wang and C. H. J. Choi, ACS Nano, 2019, 13, 5864-5884.

29 S. K. Ghosh and T. Pal, Chem. Rev., 2007, 107, 4797-4862.

30 V. Amendola, R. Pilot, M. A. latì, M. Frasconi and O. M. Maragò, J. Phys. Condens. Matter, 2017, 29, 1-48.

31 E. Prodan, C. Radloff, N. J. Halas and P. Norlander, Science, 2003, 302, 419-422.

32 K. H. Su, Q. H. Wei, X. Zhang, J. J. Mock, D. R. Smith and S. Schultz, Nano Lett., 2003, 3, 1087-1090.

33 A. I. Dolinnyi, J. Phys. Chem. C, 2015, 119, 4990-5001.

34 B. M. Reinhard, M. Siu, H. Agarwal, A. P. Alivisatos and J. Liphardt, Nano Lett., 2005, 5, 2246-2252.

35 P. K. Jain, W. Huang and M. A. El-sayed, Nano Lett., 2007, 7, 2080-2088.

36 X. Ben and H. S. Park, J. Phys. Chem. C, 2011, 115, 15915-15926.

37 P. K. Jain and M. A. El-Sayed, J. Phys. Chem. C, 2008, 112, 49544960.

38 Q. Xiang, Z. Li, M. Zheng, Q. Liu, Y. Chen, L. Yang, T. Jiang and H. Duan, Nanotechnology, 2018, 29, 1-7.

39 C. David, N. Guillot, H. Shen, T. Toury and M. L. De La Chapelle, Nanotechnology, 2010, 21, 1-6.

40 Ž. Krpetić, L. Guerrini, I. A. Larmour, J. Reglinski, K. Faulds and D. Graham, Small, 2012, 8, 707-714.

41 T. S. Rodrigues, A. G. M. Silva, A. B. L. De Moura, I. G. Freitas and P. H. C. Camargo, RSC Adv., 2016, 6, 62286-62290.

42 N. Liu, J. Liu, Y. Yang, S. Qiao, H. Huang, Y. Liu and Z. Kang, Dalt. Trans., 2015, 44, 7318-7323.

43 K. C. Grabar, K. J. Allison, B. E. Baker, R. M. Bright, K. R. Brown, R. G. Freeman, A. P. Fox, C. D. Keating, M. D. Musick and M. J. Natan, Langmuir, 1996, 12, 2353-2361.

44 M. S. Strozyk, D. Jimenez de Aberasturi and L. M. Liz-Marzán, Chem. Rec., 2018, 18, 807-818.

45 C. K. K. Choi, X. Zhuo, Y. T. E. Chiu, H. Yang, J. Wang and C. H. J. Choi, Nanoscale, 2017, 9, 16968-16980.

46 D. Chen, X. Zhu, J. Huang, G. Wang, Y. Zhao, F. Chen, J. Wei, Z. Song and Y. Zhao, Anal. Chem., 2018, 90, 9048-9054.

47 D. P. Cherney and J. M. Harris, Annu. Rev. Anal. Chem., 2010, 3, 277-297.

48 K. Ramser, J. Enger, M. Goksör, D. Hanstorp, K. Logg and M. Käll, Lab Chip, 2005, 5, 431-436.

49 C. B. Fox, G. A. Myers and J. M. Harris, Appl. Spectrosc., 2007,
61, 465-469.

50 M. Y. Wu, D. X. Ling, L. Ling, W. Li and Y. Q. Li, Sci. Rep., 2017, 7, $1-8$.

51 Y. Wang, K. El-boubbou, H. Kouyoumdjian, B. Sun, X. Huang and X. Zeng, Langmuir, 2010, 26, 4119-4125.

52 H. Y. Song, M. H. Ngai, Z. Y. Song, P. A. MacAry, J. Hobley and M. J. Lear, Org. Biomol. Chem., 2009, 7, 3400-3406.

53 N. G. Bastús, J. Comenge and V. Puntes, Langmuir, 2011, 27, 11098-11105.

54 D. Barriet, C. M. Yam, O. E. Shmakova, A. C. Jamison and T. R. Lee, Langmuir, 2007, 23, 8866-8875.

55 J. M. Sanderson and A. D. Ward, Chem. Commun. (Camb)., 2004, 44, 1120-1121.

56 T. H. Ha, S. O. Jung, J. M. Lee, K. Y. Lee, Y. Lee, J. S. Park and B. H. Chung, Anal. Chem., 2007, 79, 546-556.

57 C. García-Astrain, I. Ahmed, D. Kendziora, O. Guaresti, A. Eceiza, L. Fruk, M. A. Corcuera and N. Gabilondo, RSC Adv., 2015, 5, 50268-50277.

58 S. Wu, S. Y. Tan, C. Y. Ang, K. T. Nguyen, M. Li and Y. Zhao, Chem. Commun., 2015, 51, 11622-11625.

59 F. Thielbeer, K. Donaldson and M. Bradley, Bioconjug. Chem., 2011, 22, 144-150.

60 N. Sato, Y. Aoyama, J. Yamanaka, A. Toyotama and T. Okuzono, Sci. Rep., 2017, 7, 1-10.

61 P. K. Jain and M. a. El-Sayed, Chem. Phys. Lett., 2010, 487, 153164.

62 N. Guarrotxena, O. García and I. Quijada-Garrido, Sci. Rep., 2018, 8, 1-9.

63 J. H. Yoon and S. Yoon, Langmuir, 2013, 29, 14772-14778.

64 Y.-T. Yang, I.-L. Hsu, T.-Y. Cheng, W.-J. Wu, C.-W. Lee, T.-J. Li, C. I. Cheung, Y.-C. Chin, H.-C. Chen, Y.-C. Chiu, C.-C. Huang and M.Y. Liao, Anal. Chem., 2019, 91, 8213-8220.

65 C. Ma, K. Fu, M. J. Trujillo, X. Gu, N. Baig, P. W. Bohn and J. P. Camden, J. Phys. Chem. C, 2018, 122, 11031-11037.

66 O. Siiman, L. A. Bumm, R. Callaghan, C. G. Blatchford and M. Kerker, J. Phys. Chem., 1983, 87, 1014-1023.

67 A. Palm, J. Phys. Chem., 1951, 55, 1320-1324.

68 H. M. Lee, M. S. Kim and K. Kim, Vib. Spectrosc., 1994, 6, 205214.

69 S. Hong and X. Li, J. Nanomater., 2013, 2013, 1-9.

70 F. Benz, R. Chikkaraddy, A. Salmon, H. Ohadi, B. De Nijs, J. Mertens, C. Carnegie, R. W. Bowman and J. J. Baumberg, J. Phys. Chem. Lett., 2016, 7, 2264-2269.

71 B. Mir-Simon, J. Morla-Folch, P. Gisbert-Quilis, N. Pazos-Perez, H. N. Xie, N. G. Bastús, V. Puntes, R. A. Alvarez-Puebla and L. Guerrini, J. Opt., 2015, 17, 1-9.

72 M. Xiong and J. Ye, J. Shanghai Jiaotong Univ., 2014, 19, 681690.

73 C. Xie, M. A. Dinno and Y.-Q. Li, Opt. Lett., 2002, 27, 249-251.

74 W. Ji, X. Xue, W. Ruan, C. Wang, N. Ji, L. Chen, Z. Li, W. Song, B. Zhao and J. R. Lombardi, Chem. Commun., 2011, 47, 2426-2428.

75 J. Reguera, J. Langer, D. Jiménez De Aberasturi and L. M. LizMarzán, Chem. Soc. Rev., 2017, 46, 3866-3885.

76 S. Laing, L. E. Jamieson, K. Faulds and D. Graham, Nat. Rev. Chem., 2017, 1, 1-20.

77 M. Navas-Moreno, M. Mehrpouyan, T. Chernenko, D. Candas, M. Fan, J. J. Li, M. Yan and J. W. Chan, Sci. Rep., 2017, 7, 1-10.

78 F. Lussier, T. Brulé, M. Vishwakarma, T. Das, J. P. Spatz and J. F. Masson, Nano Lett., 2016, 16, 3866-3871.

79 O. Tokel, F. Inci and U. Demirci, Chem. Rev., 2014, 114, 57285752 . 
